\title{
TEM and Atom Probe Investigation of Calcium Carbonate Precipitation in Seawater
}

\author{
S.G. McMurray,* B.P. Gorman* and D. Diercks** \\ * Department of Metallurgical and Materials Engineering, Colorado Center for Advanced Ceramics, \\ Colorado School of Mines, 1500 Illinois St., Golden, CO 80401 \\ ** Department of Materials Science, University of North Texas, Denton, TX 76203
}

Upper ocean waters experience a rise in $p \mathrm{CO}_{2}$ as it simultaneously rises in the atmosphere. The result is a decrease in the oceanic concentration of carbonate ions. This, in turn, leads to a decrease in the saturation state of seawater with respect to carbonate minerals [1]. $\mathrm{CaCO}_{3}$ is not well understood regarding both precipitation and dissolution reaction kinetics and solubility behavior. In the last 50 years, it has been observed that aragonite precipitation acts in an unpredictable manner in relation to simple thermodynamic models [2]. This unpredictability holds true in both the natural and laboratory marine environments. Indirect evidence, obtained through Quartz Crystal Microbalance, suggests that there may be a surface phase with different properties of solubility, causing this unusual behavior.

In order to investigate this behavior, calcite and aragonite substrates were soaked for various times in different saturations of seawater. SEM analysis of the resulting precipitations shows an evolution of carbonate growth morphology that does not follow the Terrace Ledge Kink model for crystal growth [3]. Previous work shows that there appears to be an amorphous calcium carbonate (ACC) surface layer nm's thick above the overgrowth; and that the overgrowth itself seems to be growing epitaxially to the substrate [4].

Both TEM and SEM analysis have also revealed $\mathrm{CaCO}_{3}$ 's extreme sensitivity to beam damage. While certain techniques may mitigate this problem, it is evident that additional solutions should be pursued to more explicitly reveal composition. This paper appraises the possibility of using atom probe tomography as one such solution.

FIB/SEM was used to prepare site-specific specimens for cross-correlative atom probe and TEM analyses (Figures 1 and 2). Low angle and low current FIB specimen preparation was then combined with minimum laser pulses in the atom probe to minimize damage to the specimen surface. Results from this initial experiment on field evaporating $\mathrm{CaCO}_{3}$ are promising, though they reveal that much research still needs to be done to optimize atom probe experimental parameters. A representative mass spectrum (Fig. 3) clearly shows the presence of thermal tails, which are most likely due to excess heat buildup in the specimen during laser pulsing. This excess thermal energy is also most likely to the cause of inaccurate stoichiometry in the bulk composition analyses. Using a laser pulse energy of $0.1 \mathrm{~nJ}$, a temperature of $20 \mathrm{~K}$ the concentrations of each element are as follows: Ca: $20 \%$, C: $11 \%$, and O: at $69 \%$, whereas the theoretical composition is $20 \%, 20 \%$ and $60 \%$ respectively. We suggest that a significantly lower laser power or a longer laser wavelength should be utilized.

Investigating the intriguing problem of running an insulator in an atom probe, in addition to the unusual precipitation behavior of $\mathrm{CaCO}_{3}$, furthers the limits of our current knowledge in these fields of research. 
References

[1] S.C. Doney et al., Annu. Rev. Marine Sci. (2009) 169.

[2] P.K. Weyl Proceed. Inter. Conf. Tropical Oceanography. (1965) 178

[3] I. Markov. Crystal Growth for Beginners: Fundamentals of Nucleation, Crystal Growth andEpitaxy, Singapore (2003)

[4] S.M. McMurray, B.P. Gorman, Microsc. Microanal. 16 (Suppl. 2) (2010)

[5] This work presented in fond memory of John W. Morse, Louis and Elizabeth Scherck Professor of Oceanography at Texas A\&M University. The aid of Katherine Walton and Janie Lee at TAMU is gratefully acknowledged. This research is supported by the Department of Energy under grant DE-SC000-4303.

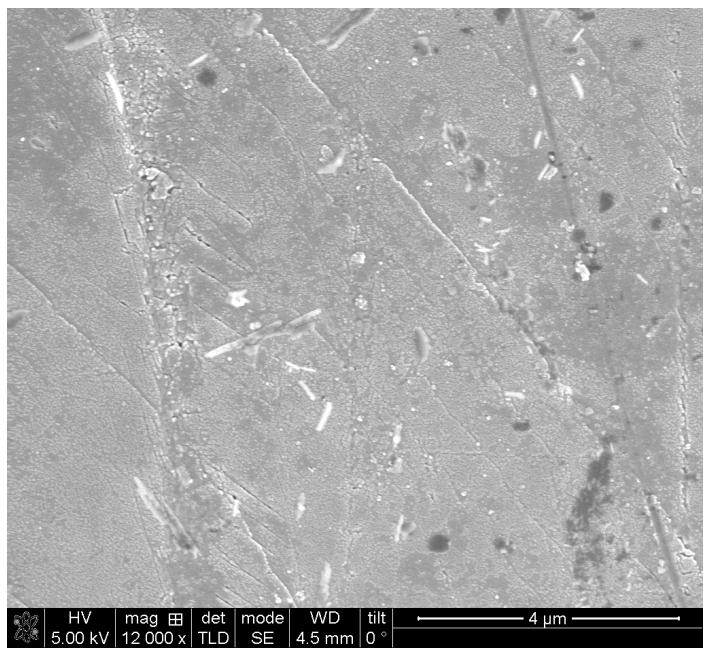

FIG. 1. SEM image of calcite surface with overgrowth sampled to make atom probe specimens.

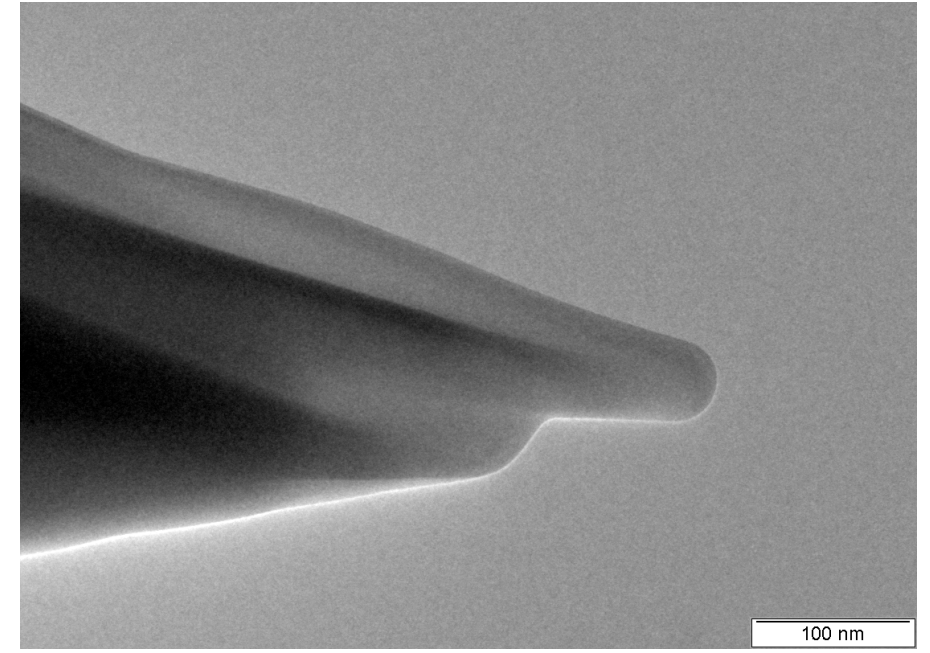

FIG. 2. TEM image of atom probe sample of calcium carbonate

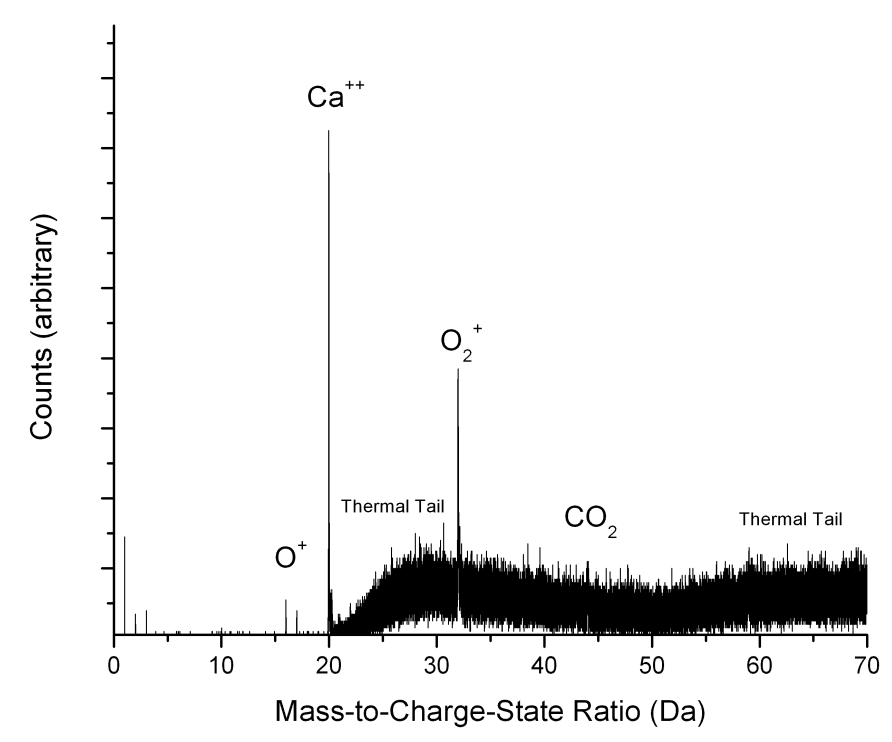

FIG. 3. Mass to charge spectrum resulting from field evaporating $\mathrm{CaCO}_{3}$ in an atom probe. 